\title{
A comparative study on the interpretation of phraseology used in histopathology reports between senders and receivers
}

\author{
M. Atchyuta ${ }^{1}$, I. V. Renuka ${ }^{2,}$, P. Prema Latha ${ }^{3}$ \\ ${ }^{\mathbf{1}}$ Assistant Professor, ${ }^{2}$ Professor, ${ }^{3}$ Professor and HOD, Dept. of Pathology, NRI Medical College, Andhra Pradesh, India
}

*Corresponding Author: I. V. Renuka

Email: repriya56@gmail.com

\begin{abstract}
Introduction: Histopathology reports should provide clinicians with diagnostically accurate and medically useful information to afford the best management to the patients.

Aim and Objectives: To make a comparative assessment of interpretation and use, of the common descriptive phrases found in histopathology reports between clinicians and pathologists, to interpret the degree of certainty among these phrases and to know the preferable phrase by clinicians and the most common phrase used by the pathologists.

Materials and Methods: A questionnaire was constructed using eight most commonly used descriptive phrases found among 1500 randomly selected histopathology reports from 2017 histopathology register, department of Pathology. This was to compare the interpretation, use and preferences of the descriptive phrases found in histopathology reports. Four groups with 25 members in each were included in the questionnaire and were asked to assign a score from 0 to 5 for these phrases to know the diagnostic level of certainty. The groups were consultant clinicians, consultant pathologists, clinical postgraduate students and pathology post graduate students. Statistical analysis was done by calculating standard deviation, student t-test and by $\mathrm{p}$ value.

Results: There was a wide variation in individual interpretation of phrases among the groups.

The preference of usage of these phrases by pathologists and by clinicians was diverse.

Conclusion: The adoption of limited number of descriptive phrases that are mutually understood and acceptable for use by both the pathologists and clinicians is recommended to avoid interpretive ambiguity in histopathology reports.
\end{abstract}

Keywords: Ambiguity, Histopathology reports, Phraseology, Questionnaire.

\section{Introduction}

Histopathology reports should provide clinicians with diagnostically accurate and medically useful information to provide best patient management. Moreover, the information should be presented in an unambiguous manner. It is common practice in the pathology community to use phrases of uncertainty in the diagnostic line. Misinterpretation of the level of diagnostic certainty may lead to inappropriate treatment. Current audit schemes in histopathology aim to provide useful reports, but do not address this problem. ${ }^{1,2}$

\section{Aim}

To make a comparative assessment of interpretation and use, of the common descriptive phrases found in histopathology reports between clinicians and pathologists, to interpret the degree of certainty among these phrases and to know the preferable phrase by clinicians and the most common phrase used by the pathologists.

\section{Materials and Methods}

A survey was conducted in the context of surgical pathology reports, based on a questionnaire which was constructed using eight most commonly used descriptive phrases. These eight descriptive phrases were chosen from 1500 randomly selected histopathology reports from 2017 January to August, histopathology register, department of Pathology, NRI Medical College. The phrases were: "Diagnostic of", "That of", "Consistent with", "Suggestive of", "Suspicious of", "Favor of", "Cannot rule out "and "Characteristic of".

Four groups with 25 members in each group were surveyed. The groups were: Consultant clinicians, Consultant pathologists, Clinical post graduate students and Pathology post graduate students. All the four group respondents were asked to estimate the "degree of certainty" for these phrases by assigning a score to each phrase on a scale from score 0 (conveying total diagnostic uncertainty) to Score 5 (conveying total diagnostic certainty). In addition, for each phrase, the consultant pathologists were asked to comment on their frequency of usage in three categories (common, uncommon, never) and the consultant clinicians were asked to comment on their preferences in two categories (like, dislike). The questionnaire used for this survey is included.

The survey was conducted partly online. A total of 100 responses were received. Statistical analysis was done by calculating standard deviation, Student's $t$-test and by calculating the probability value.

\section{Results}

For statistical analysis the respondents were divided into the following groups:

Group 1: Consultant clinicians

Group 2: Consultant pathologists

Group 3: Clinical post graduate students

Group 4: Pathology post graduate students 
The distribution of the scores for each phrase among different groups is shown in tables 1 and 2 .

The phrase "Diagnostic of" was given the highest score by both the consultant clinicians and consultant pathologists. (Groups 1 and 2) [Table 1]

The phrase "Consistent with" was given the highest score by clinical postgraduate students and the pathology post graduate students has given the high score for "Diagnostic of" (groups 3 and 4) [Table 2]

The variations in the levels of certainty understood by the different groups for the different phrases are quantified by the standard deviations from the means and are shown in Table 3. The standard deviations are therefore used as a measure of ambiguity, with a lower value indicating less ambiguity of the corresponding phrase.

The phrase "diagnostic of" has least ambiguity for consultant clinicians and also for Consultant Pathologists with standard deviation of 0.20 and 0.63 respectively.

The phrases "Suspicious of" and "Consistent with" had least ambiguity among clinical and pathology postgraduate students respectively.

When comparing the phrases themselves, the phrase with most diagnostic certainty was "diagnostic of" with a standard deviation of 0.76 and therefore stands out as better at meaning the same thing to different groups.

The difference in perceiving the level of certainty for the phrases among the groups was calculated by $t$ value and whether this difference has statistical significance or not was calculated by $\mathrm{p}$ value. $\mathrm{P}$ value $<0.05$ indicates statistical significance and is represented in Table 4.
The results showed that, the phrases diagnostic of, consistent with, suggestive of and favor of showed difference in expressing and perceiving the level of certainty among group 1 and group 2 . And this degree of difference for these phrases was statistically significant with $\mathrm{p}$ value $<0.05$.

The consultant clinicians showed highest certainty for the phrase "Diagnostic of", whereas the consultant pathologists expressed certainty for "Favor of".

Among the groups $3 \& 4$, the phrases that showed difference in expressing and perceiving the level of certainty were: "That of", "Consistent with", "Suggestive of" and "Cannot rule out" with $p$ value $<0.05$. Surprisingly, the pathology postgraduate students had shown the highest certainty for the phrase "cannot rule".

The consultant pathologists' use of the phrases and the consultant clinician's preferences for these phrases is shown in table 5 which showed the phrase "diagnostic of" was the most liked phrase by consultant clinicians.

The most preferred phrases by consultant clinicians were: diagnostic of $(100 \%)$, consistent with $(88 \%)$, 'characteristic of' (84\%) and suggestive of $(76 \%)$. The pathologists usage among the phrases preferred was as follows: diagnostic of $(56 \%)$, consistent with $(88 \%)$, 'characteristic of' (20\%) and suggestive of (96\%).

These results showed that the phrase "consistent with" was accepted by both pathologists and clinicians as the most preferred phrase in usage.

However, the commonly used phrases by pathologists were as follows: suggestive of (96\%), consistent with $(88 \%)$, favor of $(88 \%)$, diagnostic of $(56 \%)$. Though the phrase diagnostic of was liked by the clinicians $(100 \%)$, in usage it was used only by $56 \%$ of the pathologists as a common term.

Table1: Distribution of scores of different phrases among group 1 (consultant clinicians) and group 2 (consultant pathologists)

\begin{tabular}{|c|c|c|c|c|c|c|c|c|c|c|c|c|}
\hline \multirow[t]{2}{*}{ Phrase } & \multicolumn{2}{|c|}{ Score 0 } & \multicolumn{2}{|c|}{ Score 1} & \multicolumn{2}{|c|}{ Score 2} & \multicolumn{2}{|c|}{ Score 3} & \multicolumn{2}{|c|}{ Score 4} & \multicolumn{2}{|c|}{ Score 5} \\
\hline & Gr 1 & Gr 2 & Gr 1 & Gr 2 & Gr 1 & Gr2 & Gr1 & Gr 2 & Gr 1 & Gr 2 & Gr 1 & Gr 2 \\
\hline Diagnostic of & & & & & & & & 2 & 1 & 4 & 24 & 19 \\
\hline That of & 1 & & 5 & 2 & 2 & 1 & 2 & 9 & 12 & 8 & 3 & 5 \\
\hline Consistent with & & & & & 1 & & 9 & 2 & 11 & 9 & 4 & 14 \\
\hline Suggestive of & 1 & & 1 & & 2 & 1 & 9 & 7 & 11 & 13 & 1 & 4 \\
\hline Suspicious of & 4 & 2 & 11 & 7 & 4 & 11 & 6 & 4 & & 1 & & \\
\hline Favor of & 3 & & 4 & 1 & 9 & 6 & 6 & 4 & 3 & 10 & & 4 \\
\hline Cannot rule out & 5 & 5 & 12 & 7 & 3 & 9 & 3 & 3 & 2 & & & 1 \\
\hline Characteristic of & 2 & & 3 & 1 & 2 & 4 & 5 & 4 & 8 & 10 & 5 & 6 \\
\hline
\end{tabular}

Table 2: Distribution of scores of different phrases among group 3 (Clinical post graduate students) and group 4 (Pathology post graduate students)

\begin{tabular}{|c|c|c|c|c|c|c|c|c|c|c|c|c|}
\hline \multirow[t]{2}{*}{ Phrase } & \multicolumn{2}{|c|}{ Score 0 } & \multicolumn{2}{|c|}{ Score 1} & \multirow{2}{*}{\begin{tabular}{|c|} 
Score 2 \\
Gr 3
\end{tabular}} & \multirow[b]{2}{*}{ Gr 4} & \multicolumn{2}{|c|}{ Score 3} & \multicolumn{2}{|c|}{ Score 4} & \multicolumn{2}{|c|}{ Score 5} \\
\hline & Gr 3 & Gr 4 & Gr 3 & Gr 4 & & & Gr 3 & Gr 4 & Gr 3 & Gr 4 & Gr 3 & Gr 4 \\
\hline Diagnostic of & & & & & 1 & 2 & 3 & 2 & 11 & 6 & 10 & 15 \\
\hline That of & & 2 & 2 & 5 & 5 & 9 & 5 & 2 & 11 & 5 & 2 & 2 \\
\hline Consistent with & & & 1 & 1 & 3 & 7 & 7 & 14 & 13 & 31 & 1 & \\
\hline Suggestive of & 1 & & & 1 & 2 & 5 & 12 & 10 & 10 & 9 & & \\
\hline Suspicious of & & 1 & 5 & 10 & 18 & 1 & 2 & 9 & & 4 & & \\
\hline Favor of & & 1 & 2 & 7 & 11 & 2 & 8 & 9 & 4 & 5 & & 7 \\
\hline Cannot rule out & 1 & 2 & 15 & 4 & 8 & 3 & 1 & 8 & & 4 & & 4 \\
\hline Characteristic of & & & 1 & 1 & 2 & 1 & 2 & 3 & 8 & 10 & 12 & 10 \\
\hline
\end{tabular}


Table 3: Standard deviations for the interpretation of phrases describing uncertainty among the groups

\begin{tabular}{|l|c|c|c|c|c|}
\hline \multicolumn{5}{|c|}{ Phrase used } & \multicolumn{5}{|c|}{$\begin{array}{c}\text { Standard deviations } \\
\text { Clinicians }\end{array}$} & $\begin{array}{c}\text { Consultant } \\
\text { pathologists }\end{array}$ & Clinical pgs & Pathology Pgs & Overall \\
\hline Diagnostic of & 0.20 & 0.63 & 0.82 & 0.95 & 0.76 \\
\hline That of & 1.51 & 1.12 & 1.13 & 1.44 & 1.36 \\
\hline Consistent with & 0.79 & 0.65 & 0.91 & 0.72 & 0.99 \\
\hline Suggestive of & 1.09 & 0.76 & 0.91 & 0.86 & 0.94 \\
\hline Suspicious of & 1.05 & 0.96 & 0.53 & 1.26 & 1.00 \\
\hline Favor of & 1.19 & 1.15 & 0.87 & 1.33 & 1.23 \\
\hline Cannot rule out & 1.19 & 1.19 & 0.64 & 1.53 & 1.31 \\
\hline Characteristic of & 1.57 & 1.15 & 1.13 & 1.04 & 1.28 \\
\hline
\end{tabular}

Table 4: Difference in perceiving the level of certainty for the phrases

\begin{tabular}{|c|c|c|c|c|c|c|}
\hline \multirow[b]{2}{*}{ Phrases used } & \multicolumn{2}{|c|}{ Clinicians } & \multicolumn{2}{|c|}{ Pathologists } & \multirow[b]{2}{*}{ t-value } & \multirow[b]{2}{*}{$P$ value } \\
\hline & Mean & SD & Mean & SD & & \\
\hline Diagnostic of & 4.96 & 0.20 & 4.68 & 0.63 & 2.11 & 0.04 \\
\hline That of & 3.12 & 1.51 & 3.52 & 1.12 & -1.06 & 0.29 \\
\hline Consistent with & 3.72 & 0.79 & 4.48 & 0.65 & -3.71 & 0.0005 \\
\hline Suggestive of & 3.24 & 1.09 & 3.80 & 0.76 & -2.1 & 0.04 \\
\hline Suspicious of & 1.48 & 1.05 & 1.80 & 0.96 & -1.12 & 0.26 \\
\hline Favor of & 2.08 & 1.19 & 3.40 & 1.15 & -3.98 & 0.0002 \\
\hline Cannot rule out & 1.40 & 1.19 & 1.56 & 1.19 & -0.47 & 0.63 \\
\hline \multirow[t]{2}{*}{ Characteristic of } & 3.16 & 1.57 & 3.64 & 1.15 & -1.23 & 0.224 \\
\hline & \multicolumn{2}{|c|}{ Clinical pgs } & \multicolumn{2}{|c|}{ Pathology pgs } & & \\
\hline Phrases used & Mean & SD & Mean & SD & t-value & $\mathrm{P}$ value \\
\hline Diagnostic of & 4.20 & 0.82 & 4.36 & 0.95 & -0.63 & 0.52 \\
\hline That of & 3.24 & 1.13 & 2.36 & 1.44 & 2.4 & 0.02 \\
\hline Consistent with & 3.40 & 0.91 & 2.76 & 0.72 & 2.4 & 0.02 \\
\hline Suggestive of & 3.20 & 0.91 & 3.08 & 0.86 & 2.55 & 0.01 \\
\hline Suspicious of & 1.88 & 0.53 & 2.20 & 1.26 & -1.17 & 0.25 \\
\hline Favor of & 2.56 & 0.87 & 2.52 & 1.33 & 0.12 & 0.9 \\
\hline Cannot rule out & 1.36 & 0.64 & 2.80 & 1.53 & -4.3 & 0.0001 \\
\hline Characteristic of & 4.12 & 1.13 & 4.08 & 1.04 & 0.13 & 0.89 \\
\hline
\end{tabular}

Table 5: Consultant pathologists' use of the phrases and the consultant clinician's preferences for phrases

\begin{tabular}{|l|c|c|c|c|c|}
\hline \multicolumn{1}{|c|}{ Consultant Pathologists } & \multicolumn{2}{c|}{ Consultant Clinicians } \\
\hline \multicolumn{1}{|c|}{ Phrases } & Common & Uncommon & Never & Like & Dislike \\
\hline Diagnostic of & $14(56 \%)$ & $7(28 \%)$ & $4(16 \%)$ & $25(100 \%)$ & \\
\hline That of & $11(44 \%)$ & $9(36 \%)$ & $5(20 \%)$ & $9(36 \%)$ & $16(64 \%)$ \\
\hline Consistent with & $22(88 \%)$ & $3(12 \%)$ & & $22(88 \%)$ & $3(12 \%)$ \\
\hline Suggestive of & $24(96 \%)$ & $1(4 \%)$ & & $19(76 \%)$ & $6(24 \%)$ \\
\hline Suspicious of & $10(40 \%)$ & $12(48 \%)$ & $3(12 \%)$ & $3(12 \%)$ & $22(88 \%)$ \\
\hline Favor of & $22(88 \%)$ & $2(8 \%)$ & $1(4 \%)$ & $12(48 \%)$ & $13(52 \%)$ \\
\hline Cannot rule out & $6(24 \%)$ & $11(44 \%)$ & $8(32 \%)$ & $2(8 \%)$ & $23(92 \%)$ \\
\hline Characteristic of & $5(20 \%)$ & $14(56 \%)$ & $6(24 \%)$ & $21(84 \%)$ & $4(16 \%)$ \\
\hline
\end{tabular}

\section{Discussion}

This study to our knowledge is the first in this region to assess the problems of histopathologists attempting to convey their uncertainty in pathological diagnosis to their clinical colleagues through the written word in reports. The results showed that there is a wide variation in the interpretation of phrases by pathologists and clinicians, conferring potential ambiguity to histopathology reports. Given the potential impact of this ambiguity on patient care, it is perhaps surprising that this topic has not received more attention in the literature.

It is apparent that clinicians prefer the phrase "Diagnostic of" relating to unequivocal diagnoses in order to plan patient management, whereas pathologists prefer to issue reports using less definitive phrase "Suggestive of". 
The term 'Diagnostic of' was found to be the least ambiguous in interpretation in both groups; however, only $56 \%$ of pathologists used the term commonly, despite its favor among surgeons.

There may be understandable reasons why there are differences in preference for such terms between surgeons and pathologists. Where possible, clinicians are likely to prefer unambiguous diagnoses on which to base management decisions. Pathologists may use descriptions of uncertainty to accurately reflect the level of uncertainty in a diagnosis; however the possibility has been raised that pathologists might also introduce an element of uncertainty into their reporting to minimize their own personal legal risk in relation to misdiagnosis.

Attanoos et al., studied phraseology in surgical reports and communication of uncertainty between surgeons and pathologists at the University Hospital Wales. $^{3}$ Galloway and Taiyeb examined the interpretation of phrases used to describe uncertainty amongst pathologists, other doctors, and medical students online and at the University College London Medical School. ${ }^{4}$ In both of these studies, akin to our findings, there was wide variance in the interpretation of phrases between the groups studied. They similarly concluded adoption of a limited number of descriptive phrases that are mutually understood and accepted by both pathologists and clinicians is needed to avoid ambiguity in histopathology reports. An additional study addressed the need for uniformity in reporting cancer for the British National Cancer Registry. ${ }^{5}$ In his 2000 commentary on individuality in surgical pathology, Dr. Foucar aptly concluded. "There is no place for the pathologist who expresses individuality by subjecting unsuspecting patients to uncontrolled diagnostic self-expression". ${ }^{6}$

The increasing problem of medico legal challenge to any diagnostic opinion may well induce considerable caution in the use of certain phrases in reports and this may indeed explain the pathologists' reluctance to use such a definitive term as "diagnostic of". Pathologists may not express a report in terms of highest certainty to make a qualified diagnosis for a number of reasons such as inadequate tissue, extensive artifact that makes definite interpretation impossible, nonstandard histomorphology, ambiguous Immunohistochemical stains, lack of clinical information, uncertain criteria in the literature, lack of experience with the diagnosis, and hope (however unsubstantiated) to avoid legal liability for misdiagnosis.

\section{Conclusion}

Based on the statistical analysis, the present study showed that phrases like "Diagnostic of", "Suggestive of" and "Consistent with" have to be used to convey certainty in histopathology reports.

Though the clinicians prefer the most certain phrase "diagnostic of" to be given by the pathologists in the histopathology reports, the pathologists prefer to issue the reports with diagnostically less certain term such as "suggestive of" in order to avoid legal problems. And this level of uncertainty in the reports can be minimized to certain extent by conducting regular Clinicopathologic meetings that can allow the exchange of information to take place on selected cases. Such meetings also enable the clinician to understand the source of diagnostic difficulties for the pathologist, preventing interpretive ambiguity and facilitating optimal patient management.

\section{References}

1. Ramsay AD, Gallagher PJ. Local audit of surgical pathology. Am J Surg Pathol. 1992;16:476-82.

2. Campbell F, Griffiths DFR. Quantitative audit of the content of histopathology reports. J Clin Pathol. 1994;47:360-1.

3. R.L. Attanoos, A.D. Bull, A.G. Douglas-Jones. Phraseology in pathology reports: a comparative study of interpretation among pathologists and surgeons. J Clin Pathol. 1996;49(1):79-81.

4. M. Galway, T. Taiyeb, The interpretation of phrases used to describe uncertaintyin pathology reports, Pathol. Res Int. 2011 (2011), Article 656079.

5. P. Silcocks, M. Page, What constitutes a histological confirmation of cancer? A survey of terminology interpretation in two English regions. J Clin Pathol. 2001;54(3):246-248.

6. E. Foucar. "Individuality" in the specialty of surgical pathology: self-expressionor just another source of diagnostic error? Am J Surg Pathol. 2000;24(11):15731576. 